in response, this hospice has set up a Volunteer Discharge Buddy Service to help alleviate these feelings, to maintain patient safety and restore confidence on returning home.

Aims The service aims to support patients and families with practical tasks during and following discharge. The Buddies offer support on the day of discharge and make follow-up visits for up to five weeks. They liaise with community services and signpost carers and families to other services as needed.

Methods To recruit Buddies, there is a rigorous selection process and intensive two-day training programme, which includes issues around working with palliative care patients; lone working policy; safeguarding and other relevant topics. The Discharge Coordinator allocates patients to Buddies, who meet each other prior to discharge; more complex patients are allocated two Buddies. All visits are recorded in the electronic patient notes. We hold monthly supervision sessions where the Buddies discuss their experiences and bring new ideas to develop the service. We find these invaluable.

Results An evaluation form is sent to each patient. Feedback indicates that patients and families feel very reassured by the service we provide. Feedback from colleagues has also been positive. In response to considerable interest from other hospices, we have developed an information pack to assist them in setting up their own service. Colleagues from other hospices have also visited.

Conclusion Uptake has been excellent and we are planning to recruit more Buddies and extend the service to include a 'sitting' service. Additionally, we have supported the community care teams by relieving them of the simpler issues that patients frequently raise following discharge and prevented possible re-admissions when patients or their families panicked when faced with the reality of being at home.

\section{P-178 CAN WE GIVE DYING HOSPITAL PATIENTS BETTER CARE?}

'Keri Thomas, '1ulie Armstrong Wilson, ${ }^{2}$ Collette Clifford. 'Gold Standards Framework Centre C.I.C. Shrewsbury, UK; ${ }^{2}$ Birmingham University, Birmingham, UK

\subsection{6/bmispcare-2017-hospice.203}

Aims A third of hospital patients are in their last year of life, almost $50 \%$ of people die in hospital, and most do not die where they choose. Poor end of life care in UK hospitals has received much recent attention and is recognised as a key area for improvement. With earlier recognition and a more proactive approach, more might receive better care, be discharged home earlier, with fewer hospital deaths.

The Gold Standards Framework (GSF) is a quality improvement programme delivering proactive person-centred population-based care for people in the last years of life in all settings. We report on the introduction and impact of the first eight years of the GSF Acute Hospitals Programme, with findings from the first GSF Accredited wards.

Method The structured two- year GSF Hospital training programme includes training, tools, support and evaluation at organisational, patient and staff level, including measures of progress and attainment and some independent evaluations. GSF encourages teams to identify patients earlier (aiming for the $30 \%$ figure where appropriate), assess their needs (offering all initial advance care planning discussions) and proactively planning care aligned to preferences, enabling more to die at home.

Results Evaluations indicate significant progress in several key areas in many hospitals and a 'culture change' on the wards. GSF Accredited wards show embedded good practice including earlier identification (average 35\%), more proactive needsbased approach, more offered advance care planning discussions (75\%-95\%), better communication with GPs, reduced hospitalisation and improved more appropriate referrals to palliative care specialists.

Conclusions Findings from the first phases of GSF trained hospitals and the first accredited wards show significant changes, with proactive, better coordinated, person-centred care. A key factor is earlier recognition which is increasingly being attained.

\section{P-179 IMPROVING CARE FOR PATIENTS WHO MAY BE IN THEIR LAST MONTHS OF LIFE: THE LENS OF ACUTE ADMISSIONS}

${ }^{1}$ Susanna Shouls, ${ }^{2}$ Vince Connolly, ${ }^{2}$ Gavin Eyres, ${ }^{1}$ Lesley Goodburn, ${ }^{1}$ Anita Hayes, ${ }^{1}$ Paul Hayes, 'Claire Henry. 'The National Council For Palliative Care, London, UK; ${ }^{2}$ Emergency Care Improvement Programme, NHS Improvement, London, UK

\subsection{6/bmispcare-2017-hospice.204}

Background The aim is to improve the quality of care for patients who may be in their last three months of life who attend or are admitted to hospital in an emergency. Hospitals are an important provider of care for this group of people (Clark et al., 2014). An emergency admission may indicate underlying clinical decline. Quality of experience of care varies (Office for National Statistics., 2015). Acute admission processes are not generally designed to manage care for this group (Bailey et al., 2010). This may result in unwanted inpatient stays and/or treatments (Cardona-Morrell et al., 2016) that affect the person's quality of (their remaining) life. Detail is important: 'sometimes, it's the little things that matter, and that is what you remember' (NHS Improving Quality., 2014). This is in the background of increasing demographic related demand for palliative care (Public Health England., 2015) and associated pressure on services (Lowthian et al., 2010).

Method Four acute hospital Trusts formed a quality improvement collaborative in 2016 with expert clinical, quality improvement and patient experience advice. The methods to diagnose underlying problems and facilitate acute physician engagement included: a 'patient/relative' experience walkthrough, a case file review and analysis of activity data and building on existing plans for improvement/known issues. The Trusts set their own priorities for improvement.

Results The 'walkthrough' highlighted areas for improvement, eg information, signage and mortuary visiting environments. The casefile review facilitated acute physician engagement in three Trusts. These highlighted some excellent practice, which was not consistent. Recognition of dying (69\% - average three Trusts); recognition clinical uncertainty of recovery $(53 \%$ average 3 Trusts); non-beneficial treatment $(65 \%$ average two Trusts). $60 \%$ patients who died in hospital had a prior visit to that Trust (three months) - potential missed opportunity to plan. 